\title{
Loss of Autonomy in Frail Elderly and the Cross-National Comparison of Differences in Treatment Approaches: A Common Project of the EAMA
}

\author{
Hans Jürgen Heppner • Dominique Somme • \\ Michael Denkinger • Reinhard Lindner • \\ Aimee D. Garcia • Katrin Singler \\ Published online: 3 October 2012 \\ (C) Springer Science+Business Media, LLC 2012
}

\begin{abstract}
A vignette case of an 82-year-old woman, hospitalised in a geriatric ward, was presented as part of an examination of how geriatric patients are treated in different countries and what the main divergences are. Standardised questionnaires were sent to the participants concerning different components of assessment, acceptance of geriatricians, medication, financial resources, social situations, and structures of geriatrics. In this cross-national comparison, $23 \%$ of hospitals assessed were found without their own geriatric ward and in most cases, no geriatricians were present in the emergency department. Complementary assessment tools of nutritional and neuropsychological status were found to be regularly used in practice, as well as assessment tools regarding mood, mobility and cognition. Differences were found in prescription of appropriate
\end{abstract}

For the VIIth advanced postgraduate course of the European Academy of Medicine of Ageing (EAMA), Sion, Switzerland

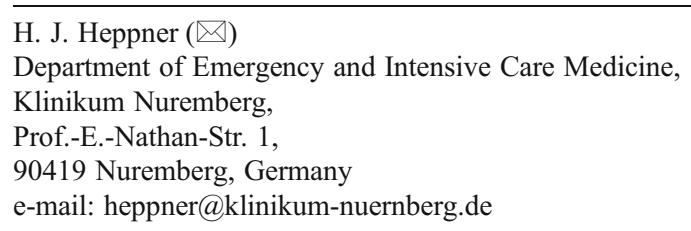

\section{H. J. Heppner $\cdot$ K. Singler}

Institute for Biomedicine of Aging,

Friedrich-Alexander-University Erlangen-Nuremberg,

Heimerichstr. 58,

90419 Nuremberg, Germany

K. Singler

e-mail: Katrin.singler@klinikum-nuernberg.de

D. Somme

Department of Geriatrics, Public Hospital Service,

Georges Pompidou European Hospital,

20-40 rue Leblanc,

75015 Paris, France

e-mail: dominique.somme@egp.aphp.fr medication, planning of ambulatory care and organisation of residential homecare, as well as problems of securing funding sources. The results show that the recognition of geriatric medicine in the different countries varies widely. Harmonising acute and long-term care for geriatric patients is only feasible with the use of international networking and common scientific projects. This underlines the importance of an institution like the European Academy of Medicine of Ageing (EAMA).

Keywords Geriatric · Cross-national · Treatment approach · European Academy of Medicine of Ageing - EAMA . Global geriatric assessment - Acute care · Long-term care · Institutional care $\cdot$ Financial sources . Health insurance law · Autonomy

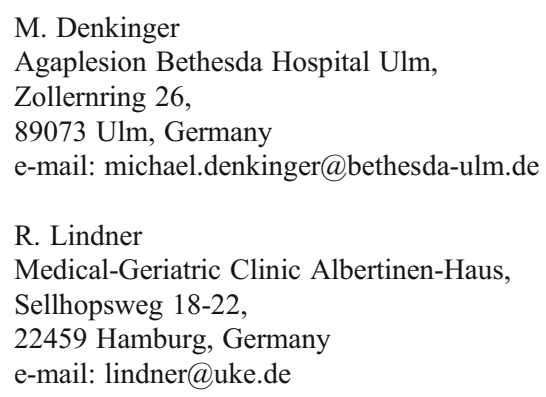

\section{A. D. Garcia}

Baylor College of Medicine, Houston VA, Medical Center, 2002 Holcombe ECL 110,

Houston, TX 77030, USA

e-mail: aimeeg@bcm.tmc.edu

K. Singler

Department of Geriatric Medicine, Klinikum Nuremberg,

Prof.-E.-Nathan-Str. 1,

90419 Nuremberg, Germany 


\section{Introduction}

Since the Consensus Conference of the European Geriatric Medicine Society (UEMS-GMS) in Malta 2008, the geriatric patient has been well defined. Globally the demographic shift shows a rapid increase in the number of geriatric patients. This implies new challenges in treatment and case management. Therefore, geriatric knowledge, special assessment and therapeutic approaches towards older adults should be implemented everywhere. As a postgraduate cohort of geriatricians, we were interested in the similarities and differences in the treatment of geriatric patients in different countries. In this common project, we wanted to point out the weaknesses in the care of older patients in a cross-national setting. This report presents the results and the interpretation of a cross-national survey in line with the postgraduate course of the European Academy of Medicine of Ageing (EAMA).

\section{Methods}

In the vignette case (this can be sent by the authors on request), an 82-year-old woman who had been hospitalised in a geriatric ward for an acute, previously unknown heart failure was presented. She was characterised as a typical geriatric patient with a state of "complete exhaustion" and a probable cognitive decline (MMSE 22/30). She was described as frail. Her previous state was that of total independence: she lived alone at home, with no external help for any dimension of functional autonomy. At discharge, the personal situation of the patient changed completely, as her condition became a challenge to herself and the caregiving team. Aim of the study was to work off the different items of assessment, to describe the acceptance of geriatricians, to prescribe medication, and to evaluate financial sources and social situation of the patient. The query was conducted during the postgraduate course of the EAMA. All participants were asked to answer the questions reffering to the vignette case and whether they run their own geriatric department or if it was combined with other medical facilities, such as internal medicine, neurology or physiatrics. It was also recorded whether a geriatrician was on duty in the emergency department $24 \mathrm{~h}$ a day, during daytime or on demand. Participants were asked to complete global geriatric and complementary assessments, as well as to report who was carrying out the assessments. Finally, another important item was the integration of geriatric medicine in the academic setting of the universities and the level of professional education in this field. In a second step, the prescription of medication, discharge planning, source funding and long-term care organisation were evaluated.

\section{Results}

Forty-five EAMA students from 19 countries (see Fig. 1) took part in the survey.

Differences were apparent right away in the results of the global geriatric assessment. The patients were assessed for cognitive disability using the Mini Mental Status Examination (MMSE) in $77 \%$ of the cases. To assess mobility, the Timed Up and Go test (TUG) was given to $46 \%$ of the participants, while $63 \%$ were assessed with a Activities of Daily Living (ADL) test and $80 \%$ were assessed with the Barthel Index as a dimension for functional independence. In more than $50 \%$ of cases, complementary assessments of nutritional status (Mini Nutritional Assessment-MNA) and
Fig. 1 Participants from this study and associated countries

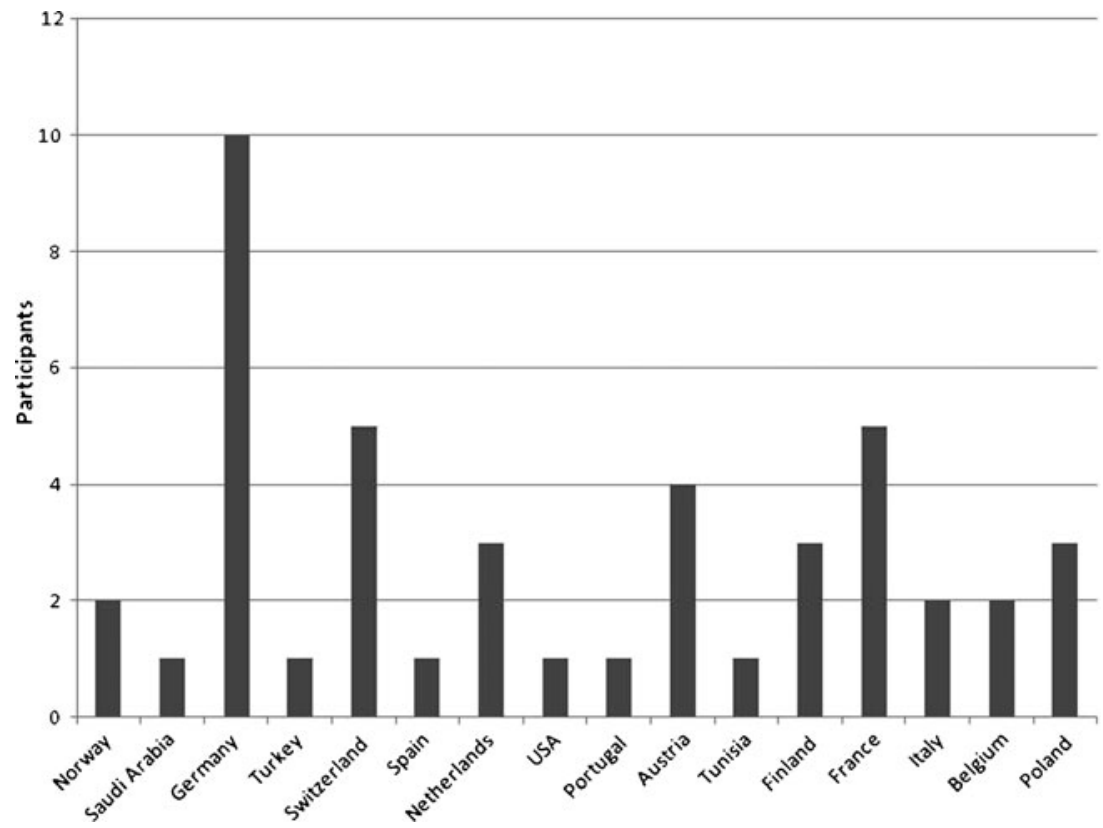


psychogeriatric status (Geriatric Depression Scale-GDS) were used. Two departments implemented used of the Resident Assessment Instrument (RAI)-emergency screener. The percentage of hospitals having no independent geriatric ward was $23 \%$, while in $94 \%$ of the hospitals, there were no geriatricians integrated in the emergency department; $30 \%$ of the hospitals had no access to a psychogeriatrician. Ranking the assessment tools according to frequency of the use of the assessments showed a decline from ADL to Barthel Index, MMSE, MNA, GDS and TUG test. European patients were much more likely to be assessed using the Barthel Index (87\% vs. $64 \%$ ). In most countries, post-acute care was covered under financial structures other than acute care. Differences were also found in prescription of appropriate medication, planning of ambulatory care or organisation of residential homecare, as well as problems of locating and securing funding sources. For the prescription of appropriate medication, national guidelines were followed and cross-national differences were identified as due to varying levels of education and as well as basic understandings of geriatric medicine.

Before proceeding with an interpretation of these results, some limitations must be considered. We had no a priori hypothesis regarding the handling of geriatric patients, but believed that specific aspects of dealing with these patients would vary across the countries (social and financial supports were expected to vary more than diagnostic approaches).

\section{Discussion}

The demographic shift is globally leading to an increase of elderly patients in the health care system, and discussions about appropriate therapy and financial disclosures, as well as prioritisation and rationing of health care expenditures on this topic are frequent. Geriatric patients commonly suffer from a combination of multiple, chronic diseases and social problems, necessitating a team approach for diagnosis and treatment. Therefore, it is indispensable for the patient to be managed by a qualified and competent physician who can provide appropriate assessment, care and follow-up. Our study was performed cross-nationally and we conducted a systematic analysis of a series of clinical and social measures to reflect the differences across countries. A crossnational comparison can help to provide a better understanding of the fragmentation of care for frail elderly patients and potentially offer possible solutions. Based on a vignette case, therapeutic approaches and medical/social supplies were compared regarding the use of assessment tools and patient access to social support. We expected our (virtual) patient to be diagnosed and assessed similarly; differences were expected regarding financial sources, health insurance and long-term care organisation. Sometimes health care services are not coordinated and some components of the system tend to work in parallel rather than in combination, with responsibilities that may overlap or leave gaps within the system. The definition of a geriatric patient and the importance of having a geriatrician in charge of care was not the same across the countries examined. Psychiatric treatment, especially psychotherapy for depression in the elderly, is still not a regular part of interdisciplinary teamwork in geriatrics. In addition, several particularly weak links in the coordination of care were identified, such as the lack of a geriatrician in the emergency department or in decisions made regarding discharge from acute care hospitals. In some countries, geriatricians that are mainly concentrated in university hospitals were found to also provide consultative care in acute hospitals, rehabilitation centers or even outreach programs in the community. It could be highlighted that there exists a big heterogeneity between the participating countries. National health insurance law is one of the biggest barriers to an implementation of the necessary steps required to improve coordination and integration of care for geriatric patients. This comparison is valuable to the determination of the status quo of treatment of and for elderly patients in a cross-national survey and could provide the basis for ongoing prospective evaluations to counter ageism. There are some hopeful signs on the horizon that all-inclusive care for geriatric patients could be achieved, but this will require an action plan starting from now. Maybe in the future, models of care can be developed to: (1) offer a complete system of primary and secondary health and social services, including acute, long-term and institutional care; (2) strengthen responsibility for the care of frail geriatric patients, and (3) implement case and public management respecting the autonomy of the elderly.

\section{Conclusion}

The countries highlighted in this article (Fig. 1) differ in many ways, historically, socially and demographically. As pointed out previously, the main value of a comparative study is to show both what constitutes effective patient care and what problems exist that prevent this achievement The results show a strong variation in the appreciation of geriatric medicine in the different countries profiled. The future challenge is in harmonising acute and long-term care for our geriatric patients.

Out of these results, the following action plan should be promoted to make standards of care more comparable before discussing the next cross-national study:

- Education in geriatrics needs be harmonised across borders

- A European exam in geriatric medicine must be required

- Geriatric medicine must have unrestricted access to academic structures 
- Professors of geriatric medicine must have the same level of education

- Geriatric medicine should be taught at all medical universities by geriatricians

This is only feasible through international networking and cooperation on shared scientific interests, conditions which underline the importance of the European Academy of Medicine of Ageing (EAMA) to the future of geriatrics.

Acknowledgements The authors thank the EAMA board members for supporting this project. Thanks also to Christina Eden and Verena Montani for assisting in the network and dealing with the correspon- dence all over the world. The authors also thank Prof. Dr. A. S. Esslinger for the health economic interpretations. H. J. Heppner is a research fellow of the "Forschungskolleg Geriatrie," Robert Bosch Foundation, Stuttgart, Germany.

Disclosure H. J. Heppner: none; D. Somme: none; M. Denkinger: reimbursement from the Robert Bosch Foundation for travel/accommodations/meeting expenses for biannual meetings; R. Lindner: none; A.D. Garcia: compensation for expert testimony as a legal consultant, payment for lectures (including service on speakers bureaus) from Celleration, Organogenesis, and Derma Sciences, and compensation for development of educational presentations from QuantiaMD; K. Singler: payment for manuscript preparation from Springer, and payment for the development of educational presentations for the online curriculum of the Robert Bosch Foundation. 